\title{
Atoms in a Radio-Frequency-Dressed Optical Lattice
}

\author{
N. Lundblad, * P. J. Lee, I. B. Spielman, B. L. Brown, W. D. Phillips, and J. V. Porto \\ Joint Quantum Institute, National Institute of Standards and Technology and University of Maryland, \\ Gaithersburg, Maryland 20899, USA
}

(Received 14 December 2007; published 14 April 2008)

\begin{abstract}
We load cold atoms into an optical lattice dramatically reshaped by radio-frequency coupling of statedependent lattice potentials. This radio-frequency dressing changes the unit cell of the lattice at a subwavelength scale, such that its curvature and topology departs strongly from that of a simple sinusoidal lattice potential. Radio-frequency dressing has previously been performed at length scales from $\mathrm{mm}$ to tens of $\mu \mathrm{m}$, but not at the single-optical-wavelength scale. At this length scale significant coupling between adiabatic potentials leads to nonadiabatic transitions, which we measure as a function of lattice depth and dressing amplitude. We also investigate the dressing by measuring changes in the momentum distribution of the dressed states.
\end{abstract}

DOI: 10.1103/PhysRevLett.100.150401

PACS numbers: $37.10 . J k, 03.75 . L m, 03.75 . \mathrm{Mn}$

For almost 20 years, optical lattices have been used to cool and confine neutral atoms, recently leading to condensed-matter-analog systems, optical clocks, and possible platforms for quantum computing [1-6]. Optical lattices are remarkably versatile, with dynamically variable depth, spin dependence, and topology [7-10]. However, they generally have a structure whose length scale is constrained by the wavelength of the lattice light. Here we demonstrate a new technique not subject to this limitation. This leads to increased flexibility in unit-cell construction, allowing access to lattice models not previously achievable. We obtain this lattice by radio-frequency (rf) dressing a spin-dependent optical lattice, in the spirit of a recentlyproposed technique [11]. The adiabatic potentials have new, subwavelength structure, which in certain limits should be ringlike within a unit cell. Extensions of the band-structure engineering demonstrated by this experiment lead to more complex lattice structures that suggest intriguing single-site wave functions and the nearestneighbor interactions required for extended BoseHubbard models [12]. The symmetry of a ringlike wave function in conjunction with the associated orbital degree of freedom can also lead to interesting condensed-matter phenomena [13].

The creation of rf-dressed adiabatic potentials in inhomogeneous magnetic fields is well-known experimentally, from the use of $\mathrm{rf}$ transitions in evaporative cooling [14] to the creation of shell-like quasi-two-dimensional systems in magnetically trapped condensates [15-17], and the use of rf dressing to effect condensate splitting and interferometry in micron-scale chip traps [18]. Optical lattices with subhalf-wavelength structure in 1D have been generated by several groups through the use of multiphoton Raman processes [19-21] or static magnetic field couplings [22]. The approach we present here, using the rf dressing of a state-dependent lattice, creates 2D subwavelength structure beyond simple lattice period division. The dressed lattice comprises unit cells whose localized adia- batic eigenstates are spatially varying superpositions of the bare spin states. We create such a dressed lattice, and load ultracold atoms into the ground band of the uppermost adiabatic potential.

For these state-dependent lattice experiments we use a spin-polarized ${ }^{87} \mathrm{Rb}\left(5^{2} S_{1 / 2}\left|F=1, m_{F}=-1\right\rangle\right)$ BoseEinstein condensate (BEC). The state dependence is produced by tuning the lattice laser between the ${ }^{87} \mathrm{Rb} D 1$ and $D 2$ resonances, where vector light shifts are important [9]. Applying an rf field of appropriate amplitude and frequency creates an adiabatic potential whose shape is distorted from the bare potentials, as shown in Fig. 1(b). After holding the atoms in the dressed lattice for a variable time, we abruptly turn off the lattice and observe the momentum distribution by imaging the atoms after time-of-flight (TOF) time $t_{\mathrm{TOF}}$. This gives information about the structure of the dressed lattice, as well as a measure of the lifetime of the dressed adiabatic eigenstates.

Our apparatus, described elsewhere [23], produces BECs of $\sim 10^{5}$ atoms in a Ioffe-Pritchard magnetic trap. We load the $m_{F}=-1$ condensate into the ground band of a 3D optical lattice via an exponentially increasing intensity ramp of duration $300 \mu \mathrm{s}(\tau=50 \mu \mathrm{s})$, a time scale adiabatic with respect to band (vibrational) excitation. We then remove the magnetic trapping fields, leaving a uniform bias field of 5.117(3) $\mathrm{mT}$ [24], a Zeeman resonance frequency $\nu_{-1,0}=36.12(2) \mathrm{MHz}$, and a quadratic Zeeman shift $\delta^{\prime} / 2 \pi=\nu_{-1,0}-\nu_{0,+1}=376(1) \mathrm{kHz}$, where $h \nu_{m, m^{\prime}}$ is the positive energy difference between Zeeman sublevels $\left|F=1, m_{F}\right\rangle$.

The lattice in the $\hat{x}-\hat{y}$ plane ( $x y$ lattice) is similar to that described in [23]. We tune a Ti:sapphire laser to $\lambda=$ $790.76 \mathrm{~nm}$, where for $\sigma^{+}$light, atoms in the three $F=1$ Zeeman sublevels $m_{F}=-1,0,+1$ experience total light shifts in the approximate ratio of $-3: 1: 5$. This can be understood as the sum of a state-independent scalar light shift and a state-dependent vector light shift - an effective magnetic field related to the local ellipticity of the optical 


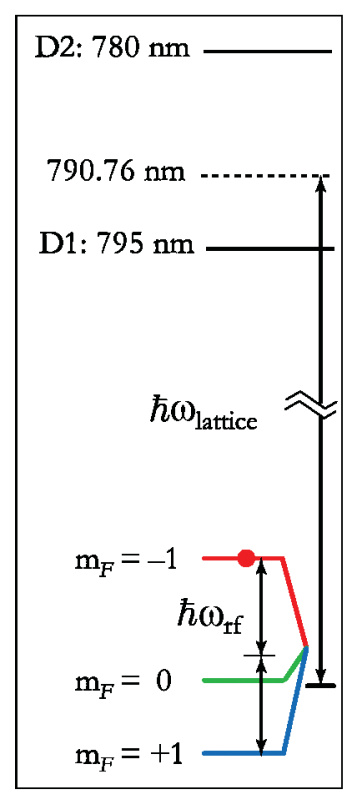

(a)

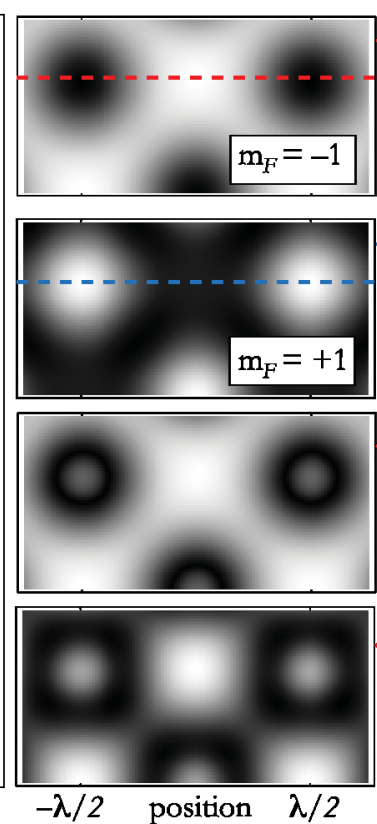

(b)

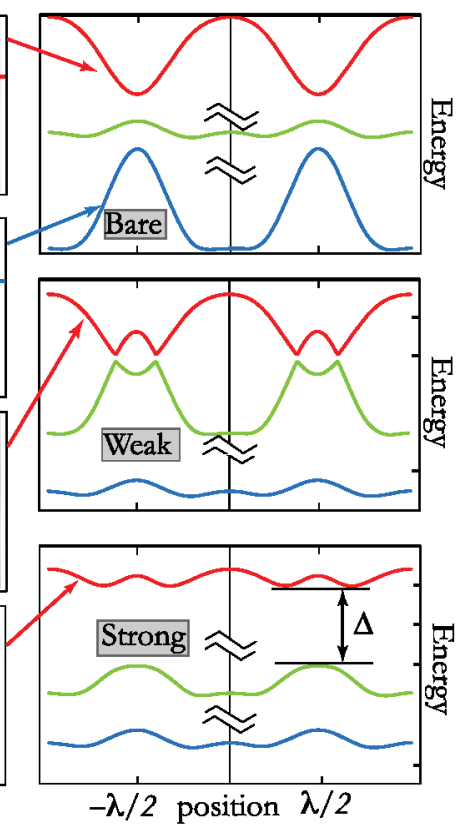

(c) polarization. For appropriate phase shifts of the lattice beams [9], the combination of the light shifts and the bias field along $(\hat{x}-\hat{y}) / \sqrt{2}$ results in the potentials shown in Fig. 1.

The atoms are confined along $\hat{z}$ (the direction of gravity) using a 1D optical lattice, derived from the same Ti:sapphire laser [25]. These lattice beams are linearly polarized, yielding an effectively blue-detuned and spinindependent lattice. As measured by pulsed-lattice diffraction [26] this vertical lattice is $6 E_{R}$ deep, where $E_{R}=$ $\hbar^{2} k^{2} / 2 M \simeq h \times 3.68 \mathrm{kHz}, k=2 \pi / \lambda$, and $M$ is the atomic mass. This lattice, together with the $x y$ lattice, fully confines the atoms in 3D, and we do not observe any displacement due to gravity over the duration of the experiment, even though the magnetic trap has been removed.

After loading, we wait $30 \mathrm{~ms}$ for the atoms to dephase and fill the lowest Bloch band of the lattice [8], such that a sudden-release TOF image will approximate the singlelattice-site momentum distribution (with resolution limited mainly by the initial spatial extent of the atom cloud). The dephasing time is well within the $1 / e$ lifetime of atoms in the lattice due to spin-flip loss from light scattering, which we measured to be $85 \mathrm{~ms}$ at an $x y$ lattice depth of $U=$ $55 E_{R}$ for $m_{F}=-1$ atoms; this lattice depth is more than twice the largest used in the experiment. The depth of the $x y$ lattice was measured with two-photon Raman vibrational spectroscopy [27].

We dress the lattice with an rf magnetic field aligned perpendicular to the bias field. The single-particle rotatingframe Hamiltonian, taking the rotating-wave approximation, is $\mathcal{H}=\mathbf{p}^{2} / 2 M+\mathcal{H}_{1}(\mathbf{r})$, where

$$
\mathcal{H}_{1}(\mathbf{r})=\left(\begin{array}{ccc}
V_{-1}(\mathbf{r})-\delta & \Omega / 2 & 0 \\
\Omega / 2 & V_{0}(\mathbf{r}) & \Omega / 2 \\
0 & \Omega / 2 & V_{+1}(\mathbf{r})+\delta+\delta^{\prime}
\end{array}\right)
$$

FIG. 1 (color). (a) The energy levels of ${ }^{87} \mathrm{Rb}$ relevant to our lattice; the rf coupling can selectively address either $m_{F}$ transition due to the quadratic Zeeman shift. (b) Top two images: the bare lattice potentials for $m_{F}= \pm 1$, exhibiting approximately opposite light shifts. Bottom two images: the uppermost adiabatic potential, weakly dressed and strongly dressed. (c) From top: bare lattice potentials for $U \simeq 10 E_{R}$; dressed adiabatic potentials at $\omega_{\mathrm{rf}} / 2 \pi=35.90 \mathrm{MHz}$, $\Omega / 2 \pi=20 \mathrm{kHz}$; dressed adiabatic potentials at $\omega_{\mathrm{rf}} / 2 \pi=35.90 \mathrm{MHz}$, $\Omega / 2 \pi=205 \mathrm{kHz}$. Note the significantly increased gap for stronger coupling. and $\delta=\omega_{\text {rf }}-2 \pi \nu_{-1,0}$. The state-dependent lattice potentials $V_{m_{F}}(\mathbf{r})$ (see Fig. 1) are calculated using our model of the lattice [23]. $\Omega$ is obtained through observations of rfdriven oscillations between $m_{F}=-1$ and $m_{F}=0$ (in a spin-independent lattice) at rates up to a maximum of $\omega_{\text {osc }} / 2 \pi=200 \mathrm{kHz} . \Omega$ differs slightly from $\omega_{\text {osc }}$ due to the multilevel nature of this system. Diagonalizing only $\mathcal{H}_{1}$ (i.e., the Born-Oppenheimer approximation) gives rise to adiabatic potentials; failure of this approximation will manifest as momentum-dependent couplings between the adiabatic potentials.

We apply rf beginning well below resonance $(\simeq-1 \mathrm{MHz})$, sweeping at constant rate $(300 \mathrm{kHz} / \mathrm{ms})$ over $\simeq 3 \mathrm{~ms}$ to a final near-resonant frequency, and hold the newly created dressed state for a variable time. The lattice beams, rf field, and bias field are then turned off in $\lesssim 1 \mu \mathrm{s}, \simeq 10 \mu \mathrm{s}$, and $\simeq 300 \mu \mathrm{s}$, respectively. After a $12.2 \mathrm{~ms}$ TOF we observe the atoms with resonant absorption imaging along the $\hat{z}$ direction.

Figure 2(a) depicts the observed width of the momentum distribution (from a Gaussian fit to the TOF density distribution) as a function of final frequency at maximum $\mathrm{rf}$ power. The optical power in the $x y$ lattice was fixed at bare depth $U \simeq 10 E_{R}$. Also shown is the expected width from the calculated ground-state momentum wave function in the adiabatic potential. The data show narrowing of the momentum distribution as the lattice is dressed, implying significant alteration of the wave function and hence the lattice structure itself. While at these dressed depths the potential exhibits ringlike character, the ground state is still simply connected: see Fig. 2(a). The observed dressed state (corresponding to $m_{F}=-1$ without rf) is predominantly a superposition of $m_{F}= \pm 1$ at these rf detunings, which we verified via Stern-Gerlach separation of the spin components during TOF. Unlike in a two-level system, the 

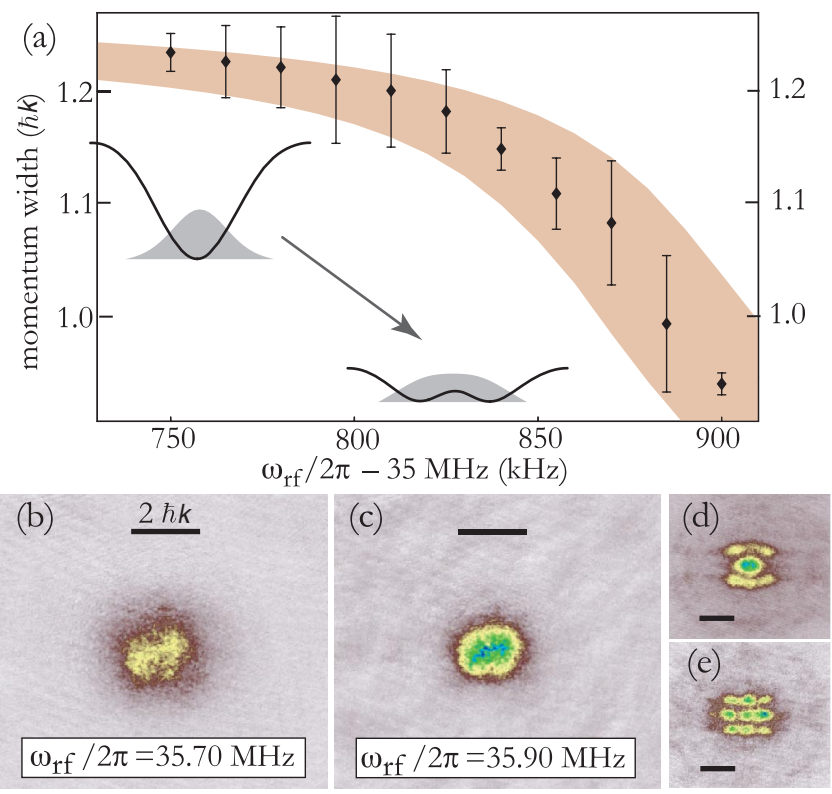

FIG. 2 (color). (a) The width of the momentum distribution as a function of final dressing frequency, as measured by absorption imaging. The width is calculated as $M d / \hbar t_{\text {TOF }}$, where $d$ is the Gaussian $1 / e$ radius along one direction, corrected for the initial cloud size. The displayed uncertainties represent $1 \sigma$ shot-to-shot scatter of five points. The shaded area represents predicted widths given the values and estimated uncertainties of the magnetic field [5.117(3) $\mathrm{mT}$ ] and the depth of the bare lattice $U=10.0(5) E_{R}$. Also shown are the bare and dressed single-site potentials (solid lines) and ground-state wave functions (gray). (b, c) Sample images of the off-resonant (effectively bare) and dressed momentum distributions, respectively, (both at $\Omega / 2 \pi=$ $205 \mathrm{kHz}$ ) showing significant narrowing of the latter. (d, e) Examples of dressed momentum distributions created through faster $(>1 \mathrm{MHz} / \mathrm{ms}) \mathrm{rf}$ ramps, such that higher vibrational states of the dressed lattice are populated.

avoided-crossing energy gap $\hbar \Delta$ between adiabatic potentials is not simply related to $\Omega$. For $\Omega \ll \delta^{\prime}, \Delta \simeq \Omega^{2} / \delta^{\prime}$, and for $\Omega \gg \delta^{\prime}, \quad \Delta \simeq \Omega / \sqrt{2}-\delta^{\prime} / 4$. For $\Omega / 2 \pi=$ $205 \mathrm{kHz}$, the largest used in this experiment, and $\omega_{\mathrm{rf}}=$ $35.90 \mathrm{MHz}$, we calculate $\Delta / 2 \pi \simeq 75 \mathrm{kHz}$, a value only weakly dependent on $U$. To further characterize our dressed lattice, we performed Raman vibrational spectroscopy, but observed that the Raman signal did not persist beyond weak dressing. This may be due to shrinking matrix elements between the combined spin and spatial eigenfunctions of the dressed bands, or increasing width of the excited bands of the (shallower) dressed lattice.

Three adiabaticity criteria are relevant to loading and holding atoms in the ground band of the dressed lattice. (1) Adiabatic following of the local spin eigenstates of $\mathcal{H}_{1}(\mathbf{r})$ during the upward rf sweep $\delta(t)$ is well satisfied. (2) Deformation of the dressed lattice with respect to vibrational excitation is nearly adiabatic, yielding predominantly ground-band occupation. Faster sweep rates yielded interesting deviations from this condition, shown in Figs. 2(d) and 2(e), in which we observe lobed structure in the momentum distributions indicating higher vibrational states. (3) The Born-Oppenheimer approximation, i.e., the degree to which zero-point motion in the lattice does not induce transitions between adiabatic potentials, is more difficult to satisfy. In previous experiments involving rf-dressed adiabatic potentials, the length scales were large enough such that this condition was easily met for cold atoms [15-18]. For our experiment, the bare lattice confinement is such that, even for our largest rf coupling, nonadiabatic loss is a factor; in our experiment these loss rates are $10^{1}-10^{3} \mathrm{~s}^{-1}$. Understanding these losses is crucial to using dressed lattices on a time scale relevant to strongly correlated many-body physics.

We load atoms into the uppermost adiabatic dressed potential using the procedure described above, and measure the number of atoms remaining in the central momentum feature, as in Figs. 2(b)-2(e), as a function of hold time. Atoms transitioning to lower adiabatic potentials appear in absorption images as high-momentum rings of $\sim 10 \hbar k$ [well outside the range of Figs. 2(b)-2(e)], and are counted as loss. Figure 3 shows loss rates as a function of calculated avoided-crossing gap $\Delta$ as a function of $U$, at dressing frequencies near $35.90 \mathrm{MHz}$. The rates are first obtained through two-parameter fits to an exponential decay and then slightly corrected via subtraction of the appropriately scaled spin-flip loss rate $\left(1 / 85 \mathrm{~ms}^{-1}\right.$ for $\left.55 E_{R}\right)$. The data are well described by $\gamma(\Delta)=A e^{-B \Delta}$. Figure 4 shows loss rate at constant $\Omega$ as a function of $U$, well described by $\gamma(U)=C e^{D U}$.

We consider our observations in the context of semiclassical theory for the losses. The traversal of a two-level avoided crossing is often described by Landau-Zener (LZ)

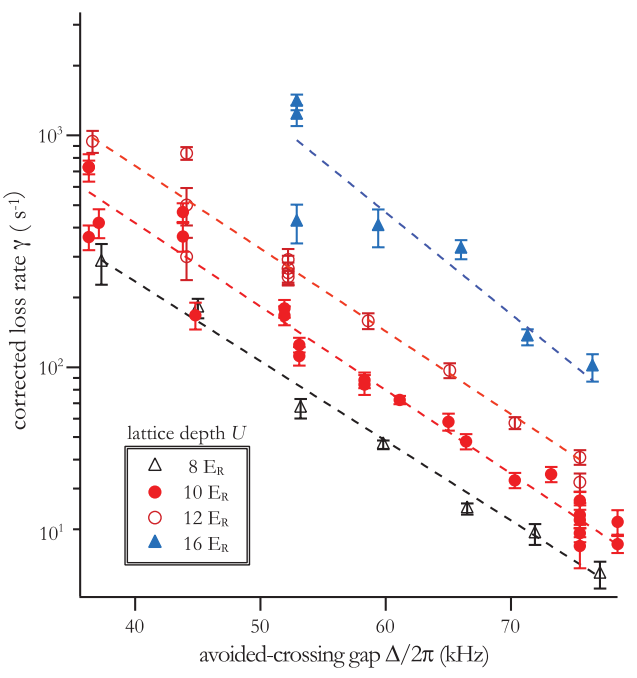

FIG. 3 (color). Dressed-state losses as a function of calculated avoided-crossing gap $\Delta / 2 \pi$ for four bare lattice depths. Vertical bars represent uncertainties of fit; multiple points near a given $\Delta$ indicate scatter in multiple runs. The data are well represented by $\gamma(\Delta)=A e^{-B \Delta / 2 \pi}$, with $B=83(6) \mu \mathrm{s}, 83(11) \mu \mathrm{s}, 82(13) \mu \mathrm{s}$, and $100(30) \mu \mathrm{s}$ for $U=8 E_{R}, 10 E_{R}, 12 E_{R}$, and $16 E_{R}$, respectively. The scaling of the data does not change if plotted versus $\Omega$. 


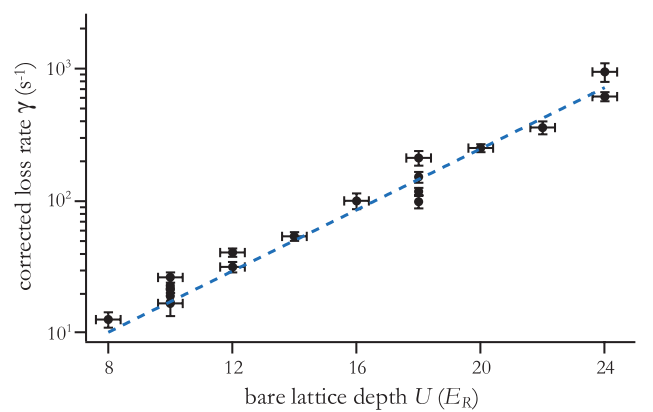

FIG. 4 (color). Dressed-state losses as a function of bare lattice depth $U$ at constant $\Omega / 2 \pi=205 \mathrm{kHz}$ and dressing frequency $35.900(35.875) \mathrm{MHz}$ for the first five (last four) depths, resulting in approximately constant gap $\Delta / 2 \pi \simeq 75 \mathrm{kHz}$. Vertical bars represent uncertainties of fit; multiple points at a given depth represent the scatter in multiple runs. Horizontal bars represent known drifts in laser power; not shown is a constant systematic uncertainty in depth determination on the order of $5 \%$. The data are well represented by a simple exponential fit $\gamma(U)=C e^{D U}$, yielding $C=1.2(4) \mathrm{s}^{-1}$ and $D=0.27(3) E_{R}^{-1}$.

theory, in which for a particle traveling along $x$ at velocity $v$ through an avoided crossing characterized by a gap $\Delta$ and bare energy levels whose difference $E(x)=E x$, the adiabatic following probability is $P_{A}=1-e^{-(1 / 2) \pi \hbar \Delta^{2} /\left(v E^{\prime}\right)}$. A semiclassical scaling argument for the motion of an atom in the ground state of a lattice site of depth $U$ yields $v \propto$ $U^{1 / 4}$ and $v E^{\prime} \propto U^{5 / 4}$, and a loss rate scaling as $\gamma \propto \omega_{l}(1-$ $\left.P_{A}\right)$, where $\omega_{l}$ is a trapping frequency and $\left(1-P_{A}\right) \ll 1$. For a particle of insufficiently large energy with respect to $\Delta$ (i.e., atoms trapped in the ground state of an adiabatic potential), the simple LZ approach may not apply. In a more sophisticated semiclassical approximation [28,29], more appropriate to ground-state trapped atoms, the resulting transition rates can be estimated as $\gamma \propto \omega_{l} e^{-\alpha \Delta / \omega_{l}}$, where $\omega_{l} \propto \sqrt{U / \Delta}$ is the trapping frequency $\left(\omega_{l} \ll \Delta\right)$ of the uppermost adiabatic potential and $\alpha \sim 1$ is a constant. The shape of our adiabatic potentials differs significantly from this simple case, but the scaling suggests transition rates that change exponentially with the coupling gap and bare lattice depth.

Unsurprisingly, the LZ model does not describe the data in Figs. 3 and 4; the exponential coefficient scales as $-\Delta$ rather than $-\Delta^{2}$ and also scales as $U$, not $-1 / U^{5 / 4}$. Fits to the LZ model are significantly worse than those in Figs. 3 and 4 , although they agree with the general trends. Similar results were found upon calculating a Fourier-weighted sum of the LZ formula over the dressed 2D ground-state wave function, $\int\left|\psi_{\mathbf{k}}\right|^{2} P_{\mathrm{NA}}(\mathbf{k}) d \mathbf{k}$. The more sophisticated semiclassical approximation performs better than the LZ approach, but still fails to satisfactorily predict the observed scaling. It is likely that $1 \mathrm{D}$ semiclassical descriptions are simply inadequate for this 2D quantum problem; perhaps a more complete solution will yield the very simple scaling we observe.

While the dressed potentials shown in Fig. 1(b) are ringlike, the corresponding ground-state wave functions are generally not. Nevertheless, ringlike wave functions with reasonable lifetimes would be possible with sufficient rf coupling; based on the data presented here, we extrapolate that ringlike wave functions of lifetime $\simeq 100 \mathrm{~ms}$ should appear for a coupling $\Omega / 2 \pi \simeq 400 \mathrm{kHz}$ and $U \simeq 55 E_{R}$.

This Letter presents observations of a novel lattice potential consisting of rf-coupled components of a statedependent optical lattice. The lifetime of this dressed lattice depends exponentially on both coupling strength and optical lattice depth. We observe lifetimes up to $100 \mathrm{~ms}$ for strongly dressed shallow lattices, a time scale sufficient for the realization of many-body condensed-matter systems.

We thank P. Zoller for inspiring this particular experiment and A. J. Daley, G. Pupillo, and P.Z. for ongoing helpful discussions. This work was partially supported by DTO and ONR. N.L., P. J.L., and B.L. B. acknowledge support from the NRC.

*nathan.lundblad@nist.gov

[1] D. Jaksch et al., Phys. Rev. Lett. 81, 3108 (1998).

[2] M. Greiner et al., Nature (London) 415, 39 (2002).

[3] D. Jaksch et al., Phys. Rev. Lett. 82, 1975 (1999).

[4] G. K. Brennen, C.M. Caves, P.S. Jessen, and I. H. Deutsch, Phys. Rev. Lett. 82, 1060 (1999).

[5] M. Anderlini et al., Nature (London) 448, 452 (2007).

[6] M. Takamoto et al., Nature (London) 435, 321 (2005).

[7] O. Mandel et al., Phys. Rev. Lett. 91, 010407 (2003).

[8] M. Greiner et al., Phys. Rev. Lett. 87, 160405 (2001).

[9] P. J. Lee et al., Phys. Rev. Lett. 99, 020402 (2007).

[10] J. Sebby-Strabley et al., Phys. Rev. Lett. 98, 200405 (2007).

[11] A. J. Daley, G. Pupillo, W. Yi, P. Zoller (to be published).

[12] V. W. Scarola, E. Demler, and S. D. Sarma, Phys. Rev. A 73, 051601 (2006).

[13] W. Liu and C. Wu, Phys. Rev. A 74, 013607 (2006).

[14] W. Ketterle and N. J. van Druten, Adv. At. Mol. Opt. Phys. 37, 181 (1996).

[15] O. Zobay and B. M. Garraway, Phys. Rev. Lett. 86, 1195 (2001).

[16] M. White et al., Phys. Rev. A 74, 023616 (2006).

[17] Y. Colombe et al., Europhys. Lett. 67, 593 (2004).

[18] T. Schumm et al., Nature Phys. 1, 57 (2005).

[19] G. Ritt et al., Phys. Rev. A 74, 063622 (2006).

[20] R. Gupta et al., Phys. Rev. Lett. 76, 4689 (1996).

[21] R. Zhang et al., Phys. Rev. A 72, 043409 (2005).

[22] D. L. Haycock et al., Phys. Rev. Lett. 85, 3365 (2000).

[23] J. Sebby-Strabley et al., Phys. Rev. A 73, 033605 (2006).

[24] Unless otherwise stated, all uncertainties herein reflect the uncorrelated combination of $1 \sigma$ statistical and systematic uncertainties

[25] The vertical lattice beams deviate from counterpropagation by $18^{\circ}$ and also differ in frequency from the 2D lattice beams by $\simeq 160 \mathrm{MHz}$.

[26] Y. B. Ovchinnikov et al., Phys. Rev. Lett. 83, 284 (1999).

[27] H. Perrin et al., Europhys. Lett. 42, 395 (1998).

[28] A. Kazantsev, G. Surdutovich, and V. Jakovlev, Mechanical Action of Light on Atoms (World Scientific, Singapore, 1990), Sec. 1.3.

[29] A. J. Daley (private communication). 